\title{
Particular Mal de Meleda Phenotypes in Tunisia and Mutations Founder Effect in the Mediterranean Region
}

\author{
Mbarka Bchetnia, ${ }^{1}$ Nadia Laroussi, ${ }^{1}$ Monia Youssef, ${ }^{2}$ Cherine Charfeddine, ${ }^{1}$ \\ Ahlem Sabrine Ben Brick, ${ }^{1}$ Mohamed Samir Boubaker, ${ }^{1}$ Mourad Mokni, ${ }^{3}$ Sonia Abdelhak, ${ }^{1}$ \\ Jameleddine Zili, ${ }^{2}$ and Rym Benmously ${ }^{4}$ \\ ${ }^{1}$ Université de Tunis El Manar, Institut Pasteur de Tunis, Laboratoire de Génomique Biomédicale et Oncogénétique (LR11IPT05), \\ BP74, 13 Place Pasteur, Belvédère, 1002 Tunis, Tunisia \\ ${ }^{2}$ Hôpital Farhat Hached, Département de Dermatologie, 4000 Sousse, Tunisia \\ ${ }^{3}$ Hôpital La Rabta, Département de Dermatologie, 1007 Tunis, Tunisia \\ ${ }^{4}$ Hôpital Habib Thameur, Département de Dermatologie, 1008 Tunis, Tunisia
}

Correspondence should be addressed to Sonia Abdelhak; sonia.abdelhak@pasteur.rns.tn

Received 30 April 2013; Accepted 5 August 2013

Academic Editor: Helmut Schöfer

Copyright ( 2013 Mbarka Bchetnia et al. This is an open access article distributed under the Creative Commons Attribution License, which permits unrestricted use, distribution, and reproduction in any medium, provided the original work is properly cited.

Mal de Meleda (MDM) is a rare, autosomal recessive form of palmoplantar keratoderma. It is characterized by erythema and hyperkeratosis of the palms and soles that progressively extend to the dorsal surface of the hands and feet. It is caused by mutations in SLURP-1 gene encoding for secreted mammalian Ly-6/uPAR-related protein 1 (SLURP-1). We performed mutational analysis by direct sequencing of SLURP-1 gene in order to identify the genetic defect in three unrelated families (families MDM-12, MDM-13, and MDM-14) variably affected with transgressive palmoplantar keratoderma. A spectrum of clinical presentations with variable features has been observed from the pronounced to the transparent hyperkeratosis. We identified the 82delT frame shift mutation in the SLURP-1 gene in both families MDM-12 and MDM-13 and the missense variation p.Cys99Tyr in family MDM-14. To date, the 82delT variation is the most frequent cause of MDM in the world which is in favour of a recurrent molecular defect. The p.Cys99Tyr variation is only described in Tunisian families making evidence of founder effect mutation of likely Tunisian origin. Our patients presented with very severe to relatively mild phenotypes, including multiple keratolytic pits observed for one patient in the hyperkeratotic area which was not previously reported. The phenotypic variability may reflect the influence of additional factors on disease characteristics. This report further expands the spectrum of clinical phenotypes associated with mutations in SLURP1 in the Mediterranean population.

\section{Introduction}

In North African populations, genetic transmission indicates a relative abundance of recessive disorders that is clearly associated with consanguineous unions [1]. Mal de Meleda (MDM; OMIM 248300) is a rare autosomal recessive skin disease with a prevalence in the general population estimated to be 1 in 100000 . Meleda refers to an Adriatic island of the Dalmatia region of Croatia, where the occurrence of the disease as an entity of its own among the various forms of Keratosis palmoplantaris hereditaria was first described [2].
The largest MDM series were reported from North Africa: Tunisia (11 families with 45 patients) and Algeria (16 families with 21 patients) $[3,4]$. MDM is characterized by erythema and hyperkeratosis of the palms and soles, extending to the dorsal aspects of the hands and feet (known as transgrediens) and perioral erythema and psoriasiform plaques on the elbows and knees. At the histological level, hyperkeratosis, hypergranulosis, and acanthosis are observed [5]. There are no specific therapies to correct the underlying genetic defect due to mutations in SLURP-1 gene located on chromosome 8q24.3. Until now, the most significant molecular advance of 
this disease has been the identification of causative mutations and the analysis of the biological role of SLURP-1 protein in the epidermis [6].

The results of previous laboratory studies showed the distribution of only four MDM mutations in North Africa p.Cys99Tyr (5 families), p.Cys77Ala (2 families), p.Gly86Arg (1 family), and $82 \mathrm{delT}$ (17 families). The mutations previously reported in Tunisian families were p.Cys99Tyr, p.Cys77Ala, and 82 delT.

Herein, we further describe three new Tunisian MDM families with the mutations 82delT and p.Cys99Tyr and presenting higher phenotypic variability.

\section{Materials and Methods}

2.1. Subjects. Three unrelated pedigrees of Tunisian descent with MDM phenotype (families MDM-12, MDM-13, and MDM-14) were referred to our laboratory to confirm diagnosis of MDM phenotype. In families MDM-12 and MDM13 , there was only one affected individual (aged of 40 years). In family MDM-14, there was 2 affected siblings that aged 2 and 13 years, respectively. The family history disclosed that the parents in families MDM-12 and MDM-14 are double and first cousins, respectively, whereas in family MDM-13, no consanguineous relationship between the parents was known. In all families, the parents were endogamous without any skin symptoms and MDM was inherited in an autosomal recessive manner (Table 1). Ethical guidelines were followed, and informed consent was obtained from all participants for the genetic investigation.

2.2. Mutational Analysis. Blood samples were drawn from each participant family member. DNA extraction from peripheral blood leucocytes was performed using standard procedures. Mutation analysis was carried out in the affected members. All exons of the SLURP-1 gene with adjacent sequences of exon-intron borders were amplified by PCR with primers and conditions described previously [4]. Mutation screening was performed by direct sequencing using Big Dye terminator technology (ABI 3130), and sequences were analyzed using Bioedit packages.

2.3. Generation of SLURP-1 Molecular Models. A model of the three-dimensional structure of SLURP-1 protein wild type and with p.Cys99Tyr variation was realized in order to assess the potential effect of p.Cys99Tyr mutation on the 3D conformation of SLURP-1 protein. In silico, modeling was performed by using I-Tasser online server [7] and PyMOL viewer [8].

\section{Results and Discussion}

\subsection{Results}

3.1.1. Clinical Findings. In family MDM-12, the patient is the offspring of parents with double consanguinity. She exhibited abnormal keratinization and yellowish erythematous lesions. The age of onset was during the first months of life. Yellow keratoderma of the palms and soles was outlined by a red scaly border in a "glove-and-socks" distribution. Hyperkeratosis spreads from the palms and soles to other parts of the body, such as dorsal aspects of the hands and feet, elbows, knees, perioral regions, and lower legs. Conical distal phalanges and nail changes including thickening of the nails were also noted. This phenotype resulted in severe functional restriction of the hands and feet (Figures 1(a) and 1(b)). In this family, two nonaffected members presented visual impairment problems. The patient in family MDM-13 presented the main clinical features of MDM without other types of complications. In both families, MDM-12 and MDM13, there was no improvement of the hyperkeratosis with age.

In family MDM-14, the two affected siblings were still young (2 and 13 years). They presented with a mild MDM phenotype characterized by slightly erythematous keratotic plaques that were light red in color and not perfectly apparent. Multiple small keratolytic pits were observed over the palmoplantar surface (Figures 1(c) and 1(d)). They consist of $2-5 \mathrm{~mm}$ pits outlined by brownish red erythema. They were variably distributed on the hyperkeratosis area and more frequently on the plantar surface. The rest of the skin was normal.

Table 2 summarizes the clinical characteristics of all the studied patients.

3.1.2. Mutational Analysis. In families MDM-12 and MDM13 , sequencing of PCR-amplified SLURP1 gene revealed the existence of a nucleotide deletion, 82delT at homozygous state in the affected individuals. This frame shift variation is leading to a creation of a premature stop codon at amino acid position 32 within exon 2 and to the synthesis of a truncated protein.

In family MDM-14, we identified a missense mutation which changes $\mathrm{G}$ to $\mathrm{A}$ at nucleotide position 297 within exon 3. It leads to an amino acid change from cysteine to tyrosine at codon 99 (p.Cys99Tyr) (Table 1).

3.1.3. Comparative Modeling of SLURP-1 Three-Dimensional Structures. Comparative modeling of SLURP-1 wild type and SLURP-1 with p.Cys99Tyr variation showed difference in the protein $3 \mathrm{D}$ conformation. The p.Cys99Tyr probably destabilizes the whole structure and particularly the third loop of the three-finger fold by changing the folding properties (Figure 2).

3.2. Discussion. The high consanguinity rates, coupled to the large family size in some communities, could induce the expression of autosomal recessive diseases, including new or very rare disorders such as MDM. Limited number of MDM cases was reported in several parts of the world [3, 4, 911]. The major reported cases of MDM in North Africa are of Tunisian descent with a total of 49 patients including the patients reported herein $[3,4,12]$. MDM is caused by mutations in SLURP-1 gene encoding for SLURP-1 protein. SLURP-1 consisted of 103 amino acids and five disulfide bridges that are critical for the correct folding and function of the protein. SLURP1 potentiates the action of acetylcholine on 


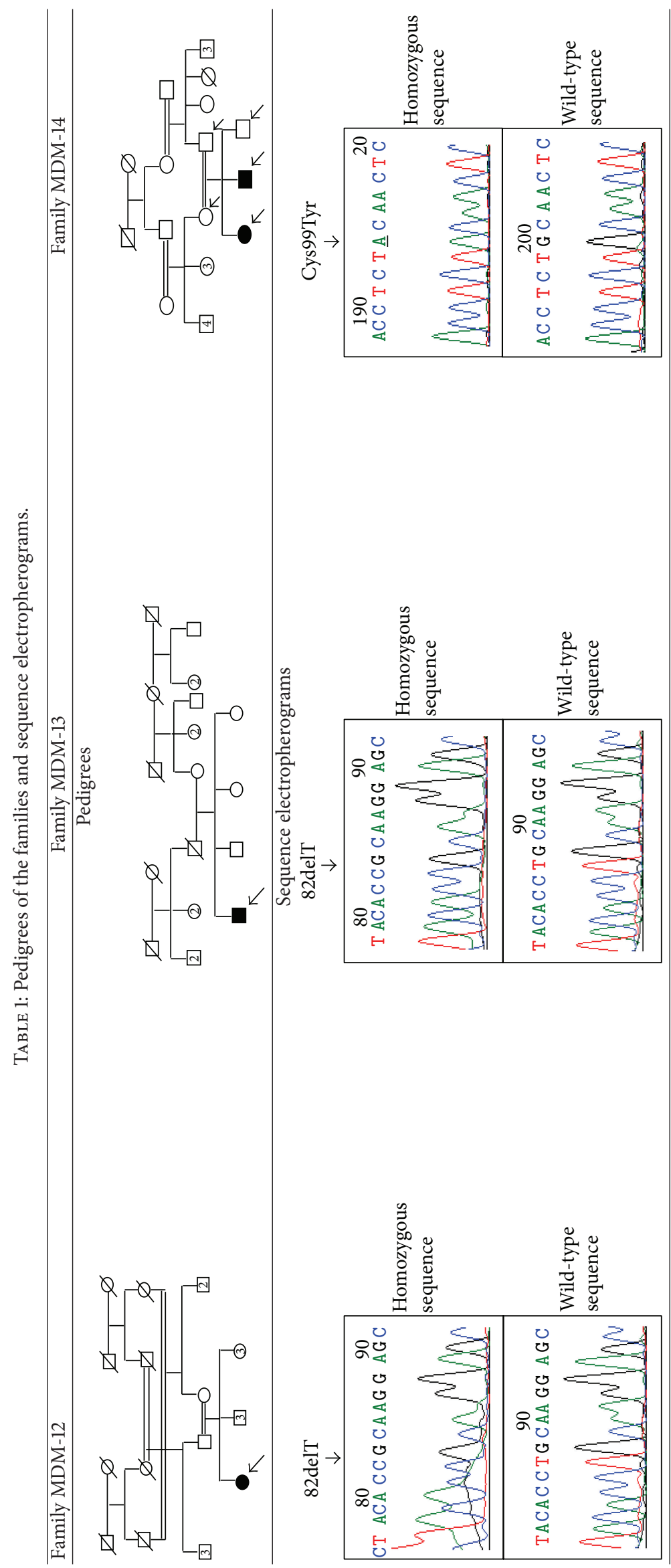




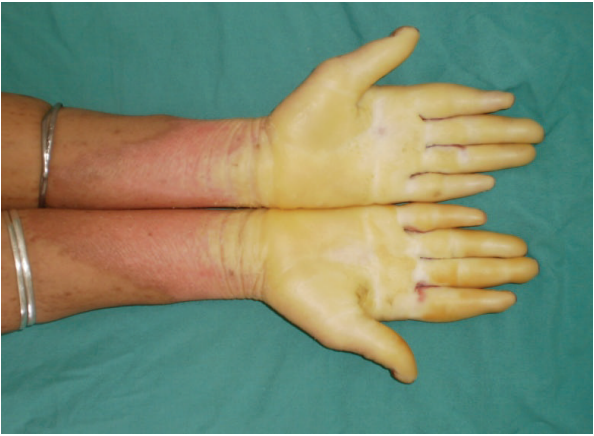

(a)

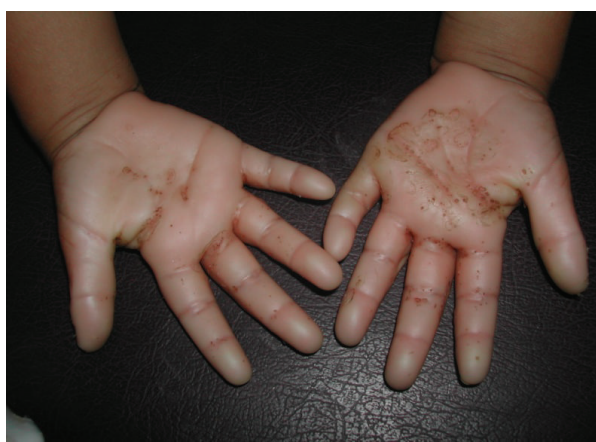

(c)

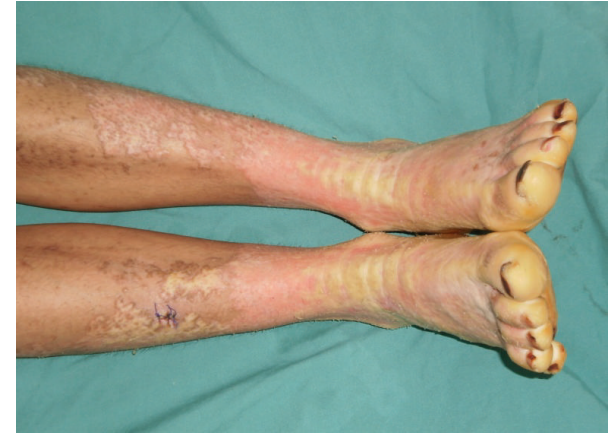

(b)

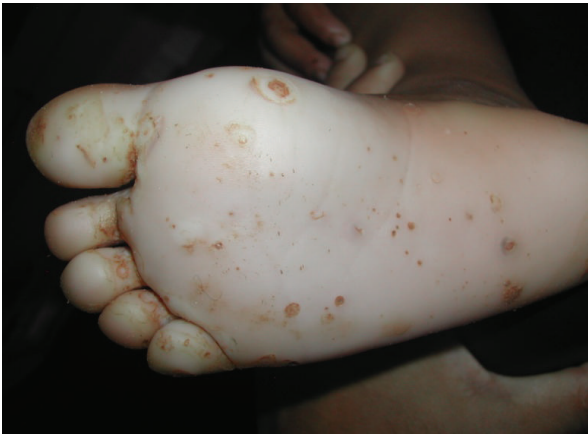

(d)

FIGURE 1: Clinical manifestations of MDM. Pronounced yellowish erythematous transgrediens palmoplantar keratoderma over the hands and feet of patient belonging to family MDM-12 ((a)-(b)). Transparent hyperkeratosis with red border delimiting the hyperkeratotic area and multiple keratolytic pits in one patient belonging to family MDM-14 ((c)-(d)).

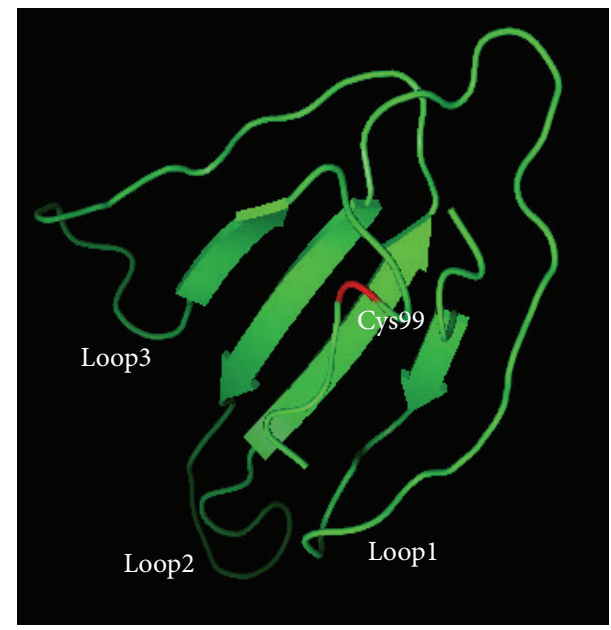

(a)

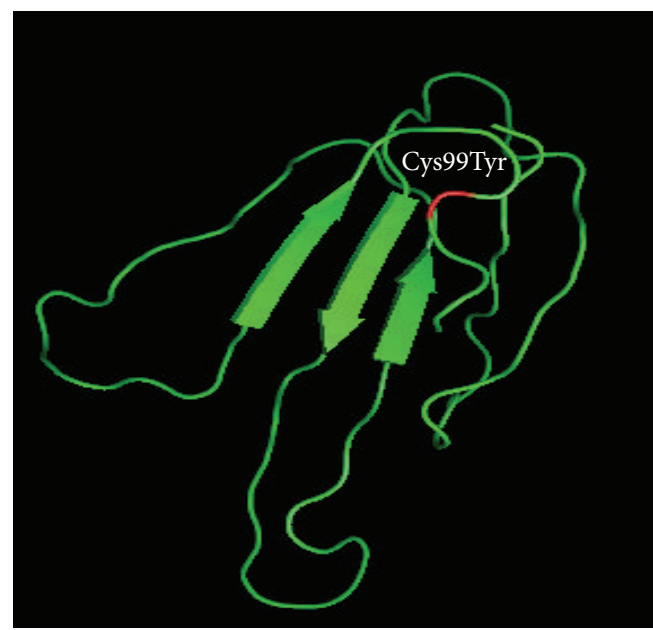

(b)

FIGURE 2: In silico modeling of the SLURP1 wild type (a) and SLURP1 with the p.Cys99Tyr mutation (b). The model shows the possible structural differences between the mutant and wild-type proteins. The presence of the p.Cys99Tyr variation led to a new protein folding missing the loops conformation. Structures are determined from amino acid sequences with I-Tasser online server and figures produced with PyMOL viewer. The p.Cys99Tyr variation position is showed by red.

the $\alpha 7$ nicotinic receptor [13], which plays an important role in the differentiation of stratified squamous epithelium $[6$, 14]. To date, only fourteen SLURP-1 mutations were described in relation with MDM phenotype. Three of them are reported in Tunisia: C77A, 82delT, and p.Cys99Tyr (Figure 3).
In this report, we further characterize new MDM cases from Tunisia. Three families were recruited with variable clinical features of MDM. We found the 82delT variation commonly in families MDM-12 and MDM-13. This frame shift mutation results in truncated, misfolded, and therefore 
TABLE 2: Clinical characteristics of the Mal de Meleda patients.

\begin{tabular}{|c|c|c|c|c|}
\hline \multirow{2}{*}{ Patient's characteristics } & \multirow{2}{*}{ Patient in family MDM-12 } & \multirow{2}{*}{ Patient in family MDM-13 } & \multicolumn{2}{|c|}{ Patients in family MDM-14 } \\
\hline & & & Patient 1 & Patient 2 \\
\hline Age (years) & 40 & 40 & 2 & 13 \\
\hline Age of onset & First months & First months & First months & First months \\
\hline Sex & $\mathrm{F}$ & M & M & $\mathrm{F}$ \\
\hline Diffuse hyperkeratosis & ++ & ++ & + & + \\
\hline Characteristics of transgressiveness & +++ & ++ & + & + \\
\hline Elbows involvement & ++ & - & - & - \\
\hline Knees involvement & ++ & - & - & - \\
\hline Palmoplantar hyperhidrosis & ++ & + & - & - \\
\hline Malodor pachyderma & +++ & ++ & + & + \\
\hline Nails involvement & +++ & ++ & ++ & ++ \\
\hline Perioral erythema & +++ & + & - & - \\
\hline Keratolytic pits & - & - & ++ & ++ \\
\hline Melanoma symptoms & - & - & - & - \\
\hline
\end{tabular}

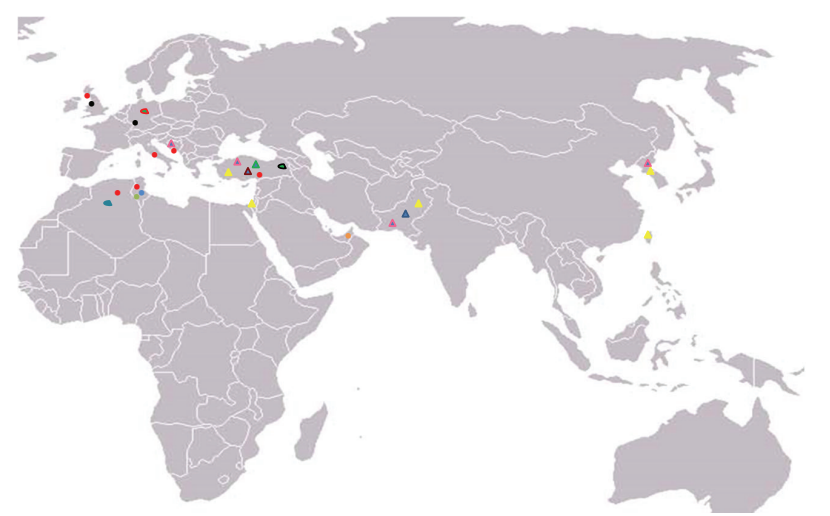

$\begin{array}{ll}\text { - Met1Lys } & \Delta \text { Ivs1 }+1 \mathrm{G}>\mathrm{A} \\ \text { - Trp15Arg } & \text { 82delT } \\ \text { Ivs2 +1G }>\text { A } & \Delta \text { Cys43Stop } \\ \text { Arg71Pro } & \text {. Cys77Ala } \\ \text { - Pro82Ser } & \Delta \text { Gly86Arg } \\ \text { A Arg96Stop } & \Delta \text { Lys98Pro } \\ \text { - Cys99Tyr } & \mathbf{\Delta} \text { Arg71His (patient origin not determined) }\end{array}$

FIGURE 3: Geographic distribution of the SLURP-1 gene reported mutations. Met1Lys (Emirates Bedouin [15]); Ivs1 + 1G > A (Pakistani [16]); Trp15Arg (German [15], Scottish [3], and Dutch [17]); 82delT (Tunisian [3], Scottish [3], Algerian [3], Croatian [9], Kurdish [10], and Italian [11]); Ivs2 + 1G > A (Algerian [3]); Cys43Stop (Turkish [18]); Arg71Pro (Dutch [17]); Cys77Ala (Tunisian [4]); Pro82Ser (Turkish [19]); Gly86Arg (Palestinian [15], Turkish [15]), and Pakistani [16]), Korean [20], Taiwanese [21]); Arg96Stop (Croatian [9], Turkish [10], Korean [20], and Pakistani [16]); Lys98Pro (Turkish [22]); Cys99Tyr (Tunisian [12]); and Arg71His (patient was reported in a study from France, and the origin is not reported [23]).

nonfunctional protein. The $82 \mathrm{delT}$ variation was previously identified across several ethnicities (Tunisian, Scottish, Algerian, Croatian, Kurdish, and Italian). It seems to be specific to the Mediterranean region (including 7 Tunisian and 10
Algerian families) suggesting the existence of a founder effect likely of Mediterranean origin where intermarriage is common [24].

The p.Cys99Tyr variation was identified in family MDM14 that originated from the South of Tunisia. It affects a cysteine implicated in one of the highly conserved disulfide bridges. Although p.Cys99Tyr is towards the end of the protein, 3D folding showed that this mutation which is within loop3 of SLURP-1 caused difference in the 3D structure compared to the wild-type SLURP-1. It was suggested that mutations involving amino acids in loop3 might affect the binding of SLURP-1 to $\alpha 7$-nAchR [23]. The p.Cys99Tyr variation was previously identified only in Tunisian families $[3,4]$. This sharp geographical demarcation is suggestive of a founder effect of Tunisian origin. Nevertheless, since some populations like Libya, Morocco, and Egypt have not yet been investigated, a more extended geographical distribution of this mutation could not be ruled out.

Comparing the disease severity between the studied families, we observed that the clinical picture varied from severe to milder transparent hyperkeratosis. The most severe phenotype was observed in family MDM-12; however, the two other patients presented a moderate phenotype. In family MDM-14, multiple keratolytic pits were observed in the hyperkeratotic area which was not previously reported. Although these unusual keratolytic pits appeared in early infancy in the palmoplantar area, the phenotype in both affected patients is currently moderate but might exacerbate with age and exposure to mechanical or heat trauma.

In the literature, The extensive scarring on the hands and feet could be associated with melanoma development. Three cases of malignant melanoma arising in the hyperkeratotic lesions of MDM have been described in the literature at late age $[21,25,26]$. One case with classical clinical MDM finding complicated by irregular hyperpigmented spots on the palmoplantar regions and the back of hands and feet was also reported [27]. We think that unfavorable environmental factors and either bacterial or fungal infection play a major 
role in the worsening of MDM and may increase the skin thickening. No melanoma symptoms were observed so far in our cases even in family MDM-12 presenting the most pronounced MDM phenotype, but it could occur at late age.

\section{Conclusion}

In this report, we further characterize new MDM cases and identified the responsible mutations. In Tunisia, the MDM patients become aware of the risk to transmit the condition to the offspring. Therefore, we believe that it is very important to inform affected families about heterozygous carriers to avoid other consanguineous marriages. While the mutational findings have improved the understanding of MDM phenotype, it is necessary to continue with therapeutic assays of SLURP-1. The function of this protein needs to be further elucidated, and therapeutic approaches for this palmoplantar keratoderma are highly required.

\section{Acknowledgments}

The authors thank the members of the studied family for their collaboration. This work was supported by the Tunisian Ministry of Public Health and the Ministry of Higher Education and Scientific Research (LR11IPT05).

\section{References}

[1] L. Romdhane and S. Abdelhak, Genetic Disorders in North African Populations, Genomics and Health in the Developing World, Oxford University Press, 2012.

[2] B. Bouadjar, S. Benmazouzia, J.-F. Prud'homme, S. Cure, and J. Fischer, "Clinical and genetic studies of 3 large, consanguineous, Algerian families with Mal de Meleda," Archives of Dermatology, vol. 136, no. 10, pp. 1247-1252, 2000.

[3] S. Marrakchi, S. Audebert, B. Bouadjar et al., "Novel mutations in the gene encoding secreted lymphocyte antigen-6/urokinasetype plasminogen activator receptor-related protein-1 (SLURP1) and description of five ancestral haplotypes in patients with Mal de Meleda," Journal of Investigative Dermatology, vol. 120, no. 3, pp. 351-355, 2003.

[4] C. Charfeddine, M. Mokni, R. Ben Mousli et al., "A novel missense mutation in the gene encoding SLURP-1 in patients with Mal de Meleda from northern Tunisia," British Journal of Dermatology, vol. 149, no. 6, pp. 1108-1115, 2003.

[5] E. Frenk, D. Guggisberg, B. Mevorah, and D. Hohl, "Meleda disease: report of two cases investigated by electron microscopy," Dermatology, vol. 193, no. 4, pp. 358-361, 1996.

[6] J. Arredondo, A. I. Chernyavsky, R. J. Webber, and S. A. Grando, "Biological effects of SLURP-1 on human keratinocytes," Journal of Investigative Dermatology, vol. 125, no. 6, pp. 1236-1241, 2005.

[7] A. Roy, A. Kucukural, and Y. Zhang, "I-TASSER: a unified platform for automated protein structure and function prediction," Nature Protocols, vol. 5, no. 4, pp. 725-738, 2010.

[8] D. Seeliger and B. L. de Groot, "Ligand docking and binding site analysis with PyMOL and Autodock/Vina," Journal of Computer-Aided Molecular Design, vol. 24, no. 5, pp. 417-422, 2010.
[9] J. Fischer, B. Bouadjar, R. Heilig et al., "Mutations in the gene encoding SLURP-1 in Mal de Meleda," Human Molecular Genetics, vol. 10, no. 8, pp. 875-880, 2001.

[10] K. M. Ward, Ö. Yerebakan, E. Yilmaz, and J. T. Çelebi, "Identification of recurrent mutations in the ARS (component $\mathrm{B}$ ) gene encoding SLURP-1 in two families with mal de Meleda," Journal of Investigative Dermatology, vol. 120, no. 1, pp. 96-98, 2003.

[11] A. Tourlaki, M. Bentivogli, V. Boneschi, and L. Brambilla, "Genetically proven Mal de Meleda complicated by Bowen's disease of the sole," European Journal of Dermatology, vol. 21, no. 2, pp. 292-294, 2011.

[12] M. Bchetnia, A. Merdassi, C. Charfeddine et al., "Coexistence of mal de Meleda and congenital cataract in a consanguineous Tunisian family: two case reports," Journal of Medical Case Reports, vol. 4, article 108, 2010.

[13] F. Chimienti, R. C. Hogg, L. Plantard et al., "Identification of SLURP-1 as an epidermal neuromodulator explains the clinical phenotype of Mal de Meleda," Human Molecular Genetics, vol. 12, no. 22, pp. 3017-3024, 2003.

[14] H. Kurzen and K. U. Schallreuter, "Novel aspects in cutaneous biology of acetylcholine synthesis and acetylcholine receptors," Experimental Dermatology, vol. 13, supplement 4, pp. 27-30, 2004.

[15] K. M. Eckl, H. P. Stevens, G. G. Lestringant et al., "MAL de Meleda (MDM) caused by mutations in the gene for SLURP1 in patients from Germany, Turkey, Palestine, and the United Arab Emirates," Human Genetics, vol. 112, no. 1, pp. 50-56, 2003.

[16] M. Wajid, M. Kurban, Y. Shimomura, and A. M. Christiano, "Mutations in the SLURP-1 gene underlie Mal de Meleda in three Pakistani families," Journal of Dermatological Science, vol. 56, no. 1, pp. 27-32, 2009.

[17] R. G. L. Nellen, M. van Geel, P. M. Steijlen, and M. A. M. van Steensel, "Compound heterozygosity for ARS component B mutations in a Dutch patient with mal de Meleda," British Journal of Dermatology, vol. 160, no. 4, pp. 878-880, 2009.

[18] M. H. Muslumanoglu, N. Saracoglu, O. Cilingir et al., "A novel mutation in the ARS (component B) gene encoding SLURP1 in a Turkish family with mal de Meleda," British Journal of Dermatology, vol. 155, no. 2, pp. 467-469, 2006.

[19] R. Gruber, H. C. Hennies, N. Romani, and M. Schmuth, "A novel homozygous missense mutation in SLURP1 causing Mal de Meleda with an atypical phenotype," Archives of Dermatology, vol. 147, no. 6, pp. 748-750, 2011.

[20] Y. J. Oh, H. E. Lee, J. Y. Ko, Y. S. Ro, and H. J. Yu, "A sporadic case of Mal de Meleda caused by gene mutation in SLURP-1 in Korea," Annals of Dermatology, vol. 23, no. 3, pp. 396-399, 2011.

[21] J.-W. Tjiu, P.-J. Lin, W.-H. Wu et al., "SLURP1 mutationimpaired T-cell activation in a family with mal de Meleda," British Journal of Dermatology, vol. 164, no. 1, pp. 47-53, 2011.

[22] Ö. Yerebakan, G. Hu, E. Yilmaz, and J. T. Çelebi, "A novel mutation in the ARS (component B) gene encoding SLURP1 in a family with Mal de Meleda," Clinical and Experimental Dermatology, vol. 28, no. 5, pp. 542-544, 2003.

[23] B. Favre, L. Plantard, L. Aeschbach et al., "SLURP1 is a late marker of epidermal differentiation and is absent in Mal de Meleda," Journal of Investigative Dermatology, vol. 127, no. 2, pp. 301-308, 2007.

[24] N. Ben Halim, N. Ben Alaya Bouafif, L. Romdhane et al., "Consanguinity, endogamy, and genetic disorders in Tunisia," Journal of Community Genetics, vol. 4, pp. 273-284, 2013. 
[25] N. Mozzillo, C. A. Nunziata, C. Caracò, F. Fazioli, and G. Botti, "Malignant melanoma developing in an area of hereditary palmoplantar keratoderma (Mal De Meleda)," Journal of Surgical Oncology, vol. 84, no. 4, pp. 229-233, 2003.

[26] L. Sartore, M. Bordignon, F. Bassetto, A. Voltan, V. Tomat, and M. Alaibac, "Melanoma in skin affected with keratoderma palmoplantaris hereditaria (Mal de Meleda): treatment with excision and grafting," Journal of the American Academy of Dermatology, vol. 61, no. 1, pp. 161-163, 2009.

[27] A. Baroni, V. Piccolo, R. di Maio, F. Romano, F. di Girolamo, and R. A. Satriano, "Mal de meleda with hyperpigmented spots," European Journal of Dermatology, vol. 21, no. 3, pp. 459-460, 2011. 


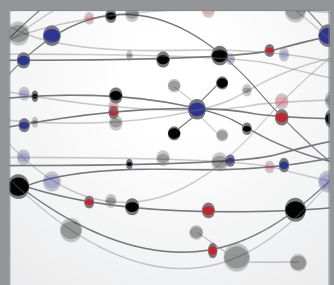

The Scientific World Journal
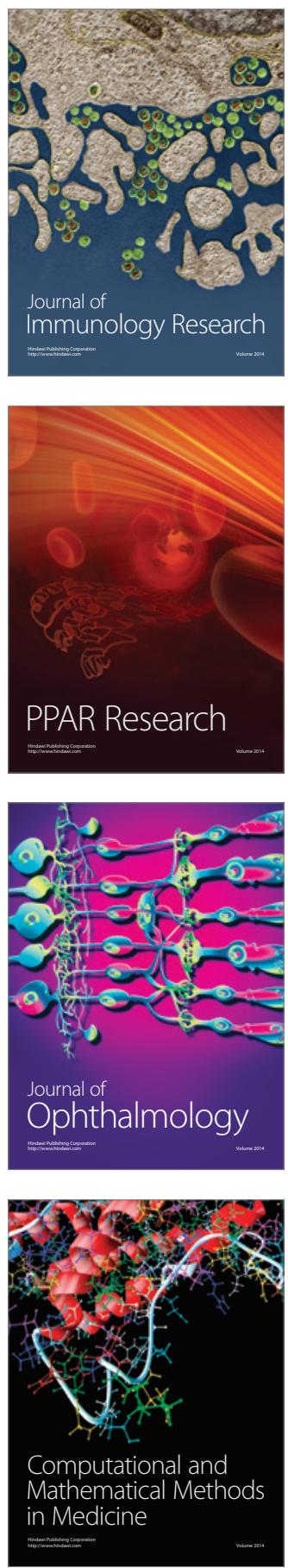

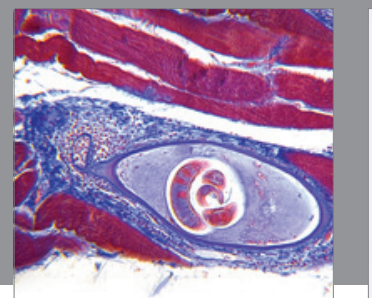

Gastroenterology

Research and Practice
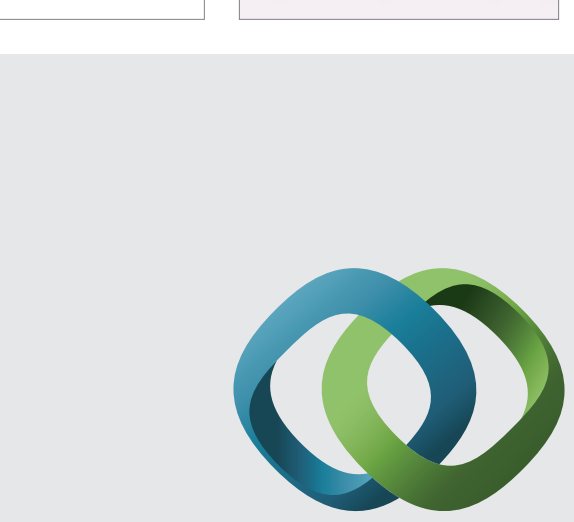

\section{Hindawi}

Submit your manuscripts at

http://www.hindawi.com
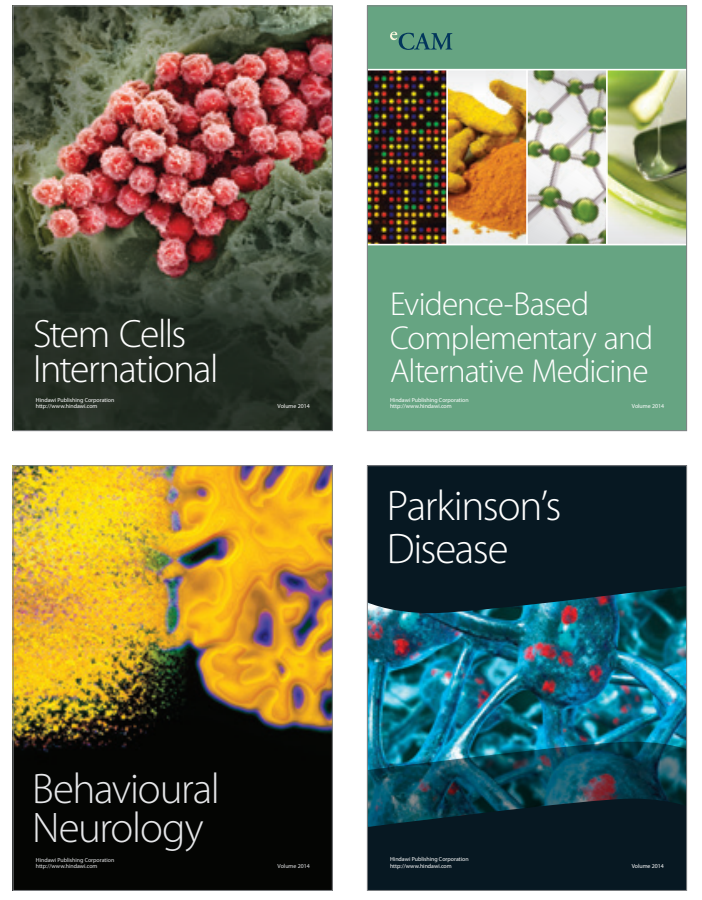
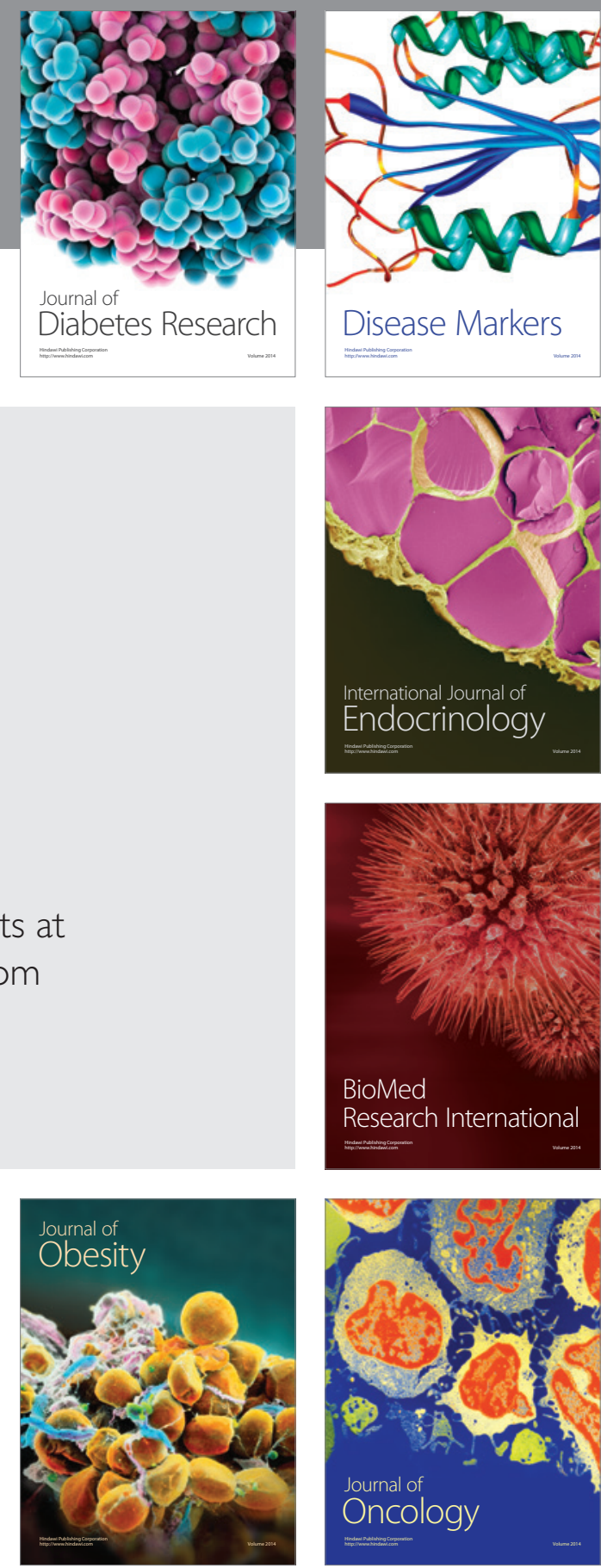

Disease Markers
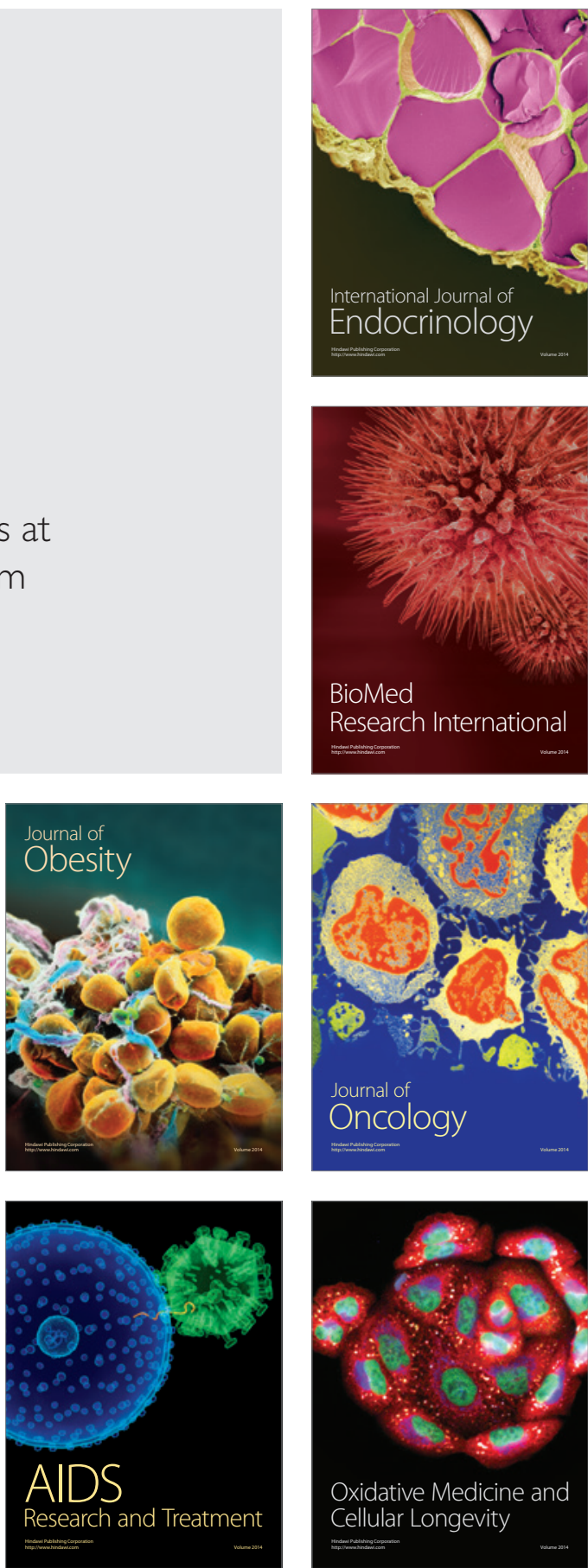